Proceedings

\title{
Demand Response Integration tEchnologies: Unlocking the Demand Response Potential in the Distribution Grid +
}

\author{
Tatiana Loureiro ${ }^{1, *}$, Raymond Sterling ${ }^{1}$ and Meritxell Vinyals ${ }^{2}$ \\ 1 R2M Solution, Calle Cervera 59, 1 D, 28033 Madrid, Spain; raymond.sterling@r2msolution.com \\ 2 CEA, LIST, Laboratoire d'Analyse des Données et d'Intelligence des Systèmes, 91191 Gif-sur-Yvette, \\ France; meritxell.vinyals@cea.fr \\ * Correspondence: tatiana.loureiro@r2msolution.com; +34-918276597 \\ † Presented at Sustainable Places 2018 (SP 2018), Aix-les Bains, France, 27-29 June 2018.
}

Published: 28 August 2018

\begin{abstract}
DRIvE is a 36 months Horizon 2020 project which main objective is to unlock the Demand Response (DR) potential of residential and tertiary buildings in the distribution grid. DRIvE comprehensive platform can be seamlessly integrated with existing assets and buildings to achieve optimal operations in the next generation of Smart Grids, paving the way to a fully deployed DR market in the distribution network.
\end{abstract}

Keywords: demand response; flexibility; distribution grid; buildings; $\mathrm{H} 2020$

\section{Introduction}

Demand Response Integration tEchnologies (DRIvE) is a Horizon 2020 EU-funded project carried out by 9 partners from across Europe that aims to unlock the demand response (DR) in the distribution grid by linking together cutting-edge science in artificial intelligence, forecasting and cyber security with emerging innovative SMEs making first market penetration in EU DR markets [1]. In doing so, near market solutions are strengthened with innovative functionalities that support a vision of an "internet of energy" and "collaborative energy network." DRIvE uses artificial intelligence to bring decentralized management and DR services to prosumers, grid stakeholders and distribution system operators.

DRIvE undermining process will consist of 3 phases: (1) unlock DR potential of buildings; (2) enable effective and secure decentralised management of the grid; and (3) make a synergistic use of validation activities to boost DRIvE development.

These overarching objectives will be achieved thanks to the development and validation of a fully-integrated ICT infrastructure consisting of interoperable DR-enabling Energy Management solutions for residential and tertiary buildings and platform for effective and secure management of flexibility at the level of the distribution grid.

The project, coordinated by the French Alternative Energies and Atomic Energy Commission (CEA), started in December 2017 and will last three years.

\section{Why DRIvE?}

It is widely recognised that increasing flexibility is key for the reliable operation of future power systems with very high penetration levels of Variable Renewable Energy Sources (VRES) [2]. Flexibility is the ability of a power system to maintain continuous service in the face of rapid and large swings in supply or demand. The most significant source of flexibility in a future scenario with 
high penetration of VRES is demand response. The new challenge is to unlock the very high potential of DR in the distribution grid where the main sources of flexibility are the residential and tertiary buildings, representing $70 \%$ of the total DR potential.

DRIvE will unlock the DR potential of residential and tertiary buildings in the distribution grid through a comprehensive platform that integrates existing assets and buildings to achieve optimal operations in Smart Grids, towards a fully deployed DR market in the distribution network.

\section{What is DRIvE?}

DRIvE is a research project that links together cutting-edge science in Multi-Agent Systems (MAS), forecasting and cyber security with emerging innovative SMEs making first market penetration in EU DR markets. In doing so, near market solutions are strengthened with lower TRL, higher risk functionalities that support a vision of an "internet of energy" and "collaborative energy network." From the research side, MAS will move closer to real time operations and progress from a limited number of assets toward decentralized management of a larger number of assets providing DR services to prosumers, grid stakeholders and distribution system operators.

\subsection{Process}

To break out of the current status quo, DRIvE will develop and validate a fully-integrated ICT infrastructure consisting of interoperable DR-enabling Energy Management solutions for residential and tertiary buildings and platform for effective and secure management of flexibility at the level of the distribution grid, as illustrated in Figure 1. The DRIvE process will consist ofs:

1. Unlock DR potential of buildings;

2. Enable effective and secure decentralised management of the grid;

3. Make a synergistic use of validation activities to boost DRIvE development.

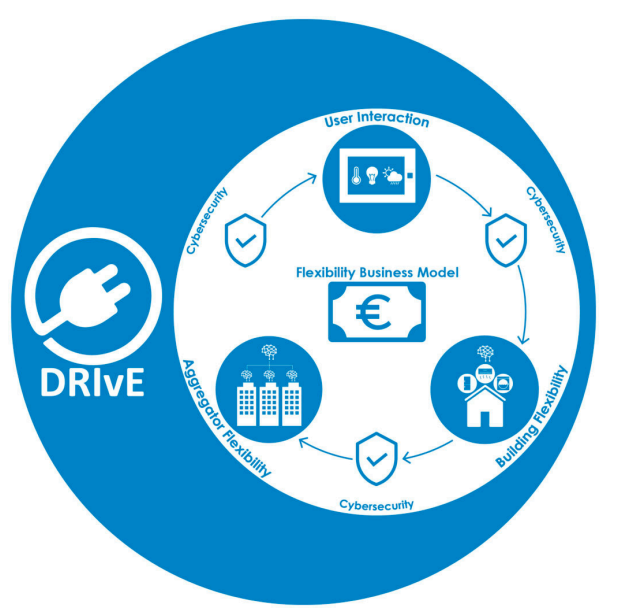

Figure 1. DRIvE solution. 


\subsection{Objectives}

- Unlock DR potential in residential and tertiary buildings through low cost solutions that are universally interoperable, integrating innovative load prediction and optimization algorithms (Figure 2)

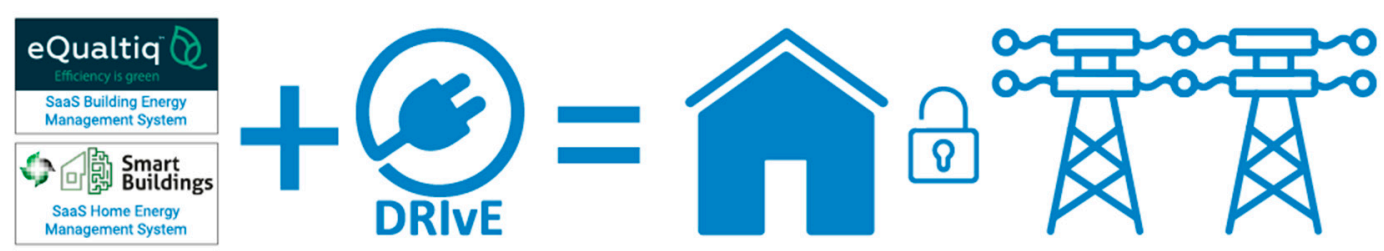

Figure 2. DRIvE unlocking DR potential.

- Optimize distribution grid flexibility through an integrated Multi-agent based Demand Response ICT platform for aggregators integrating last advances in distributed real-time control architecture, artificial intelligence and communications (Figure 3)

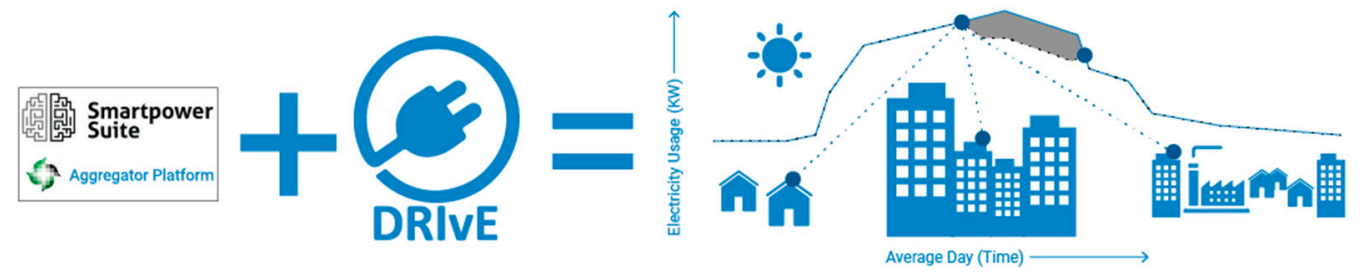

Figure 3. DRIvE flexibility optimization.

- Demonstration of secure communication through the design and development of cyber-security components for Smart Grids (Figure 4)

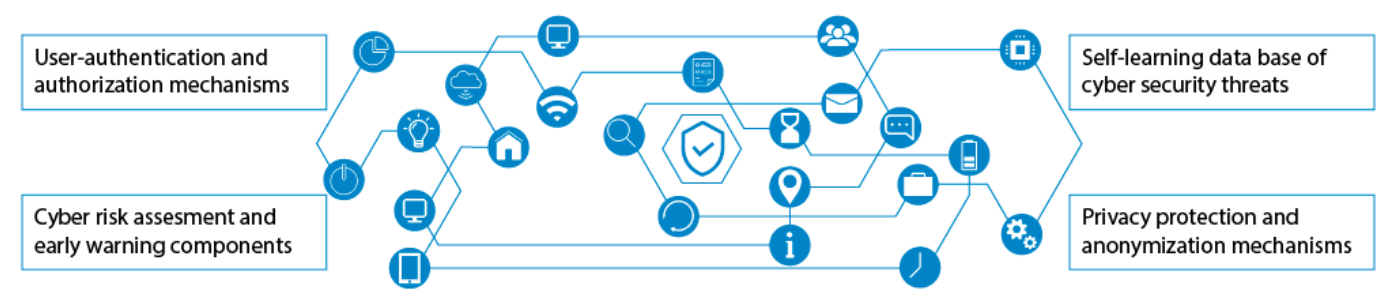

Figure 4. DRIvE cyber-security approach.

- Engage and stimulate customers to participate in DR programs through a consumer portal (Figure 5)
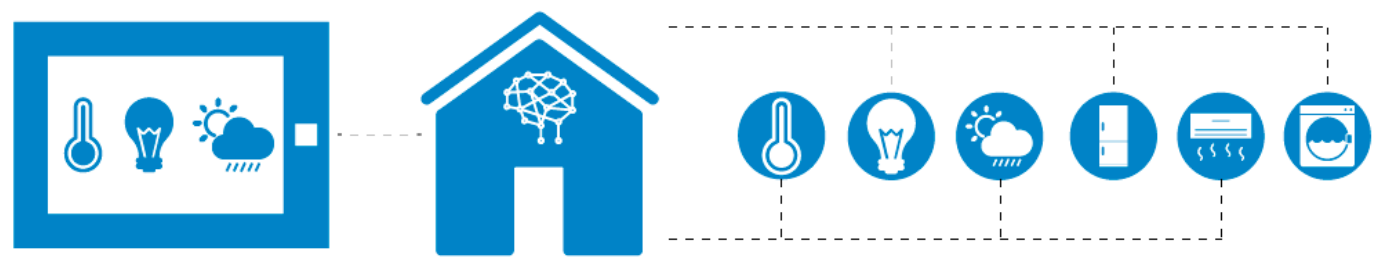

Figure 5. DRIvE consumer engagement.

\subsection{Impact of DRIvE}

DRIvE will have impacts on several areas, represented in Figure 6. 

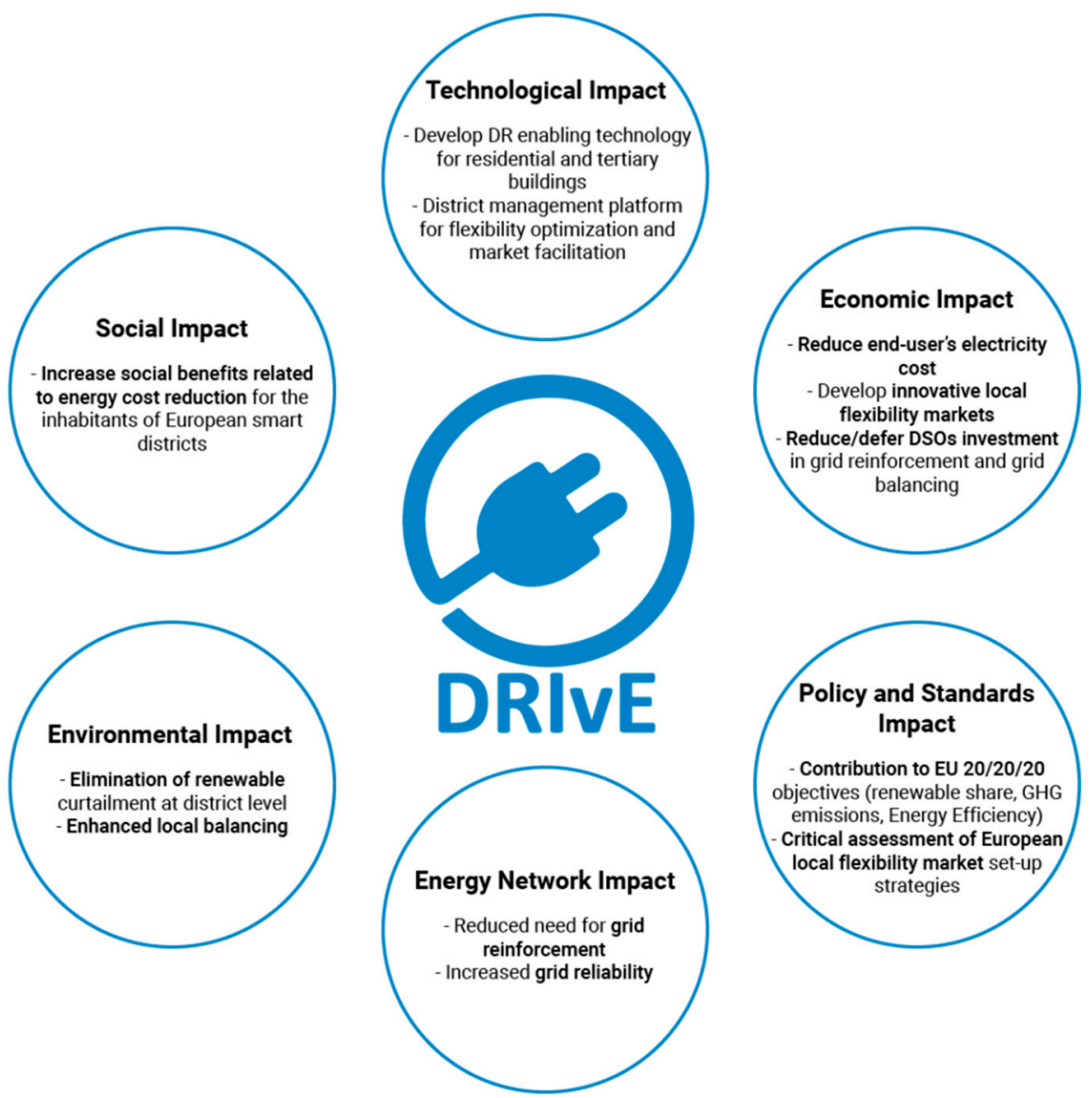

Figure 6. DRIvE Impact.

\subsection{Demo Sites}

DRIvE will demonstrate the effectiveness of the proposed flexibility management platform though a wide set of validation activities involving five pilot sites and globally covering all services within the DR value chain. The demo sites used during the project are:

1. Blaenau Gwent District (UK)

2. DEVO District (Netherlands)

3. Giessenwind wind farm (Netherlands)

4. ADO Stadium (Netherlands)

5. COMSA head office (Spain)

\subsection{Consortium}

The Consortium that constitutes DRIvE represents a multi-disciplinary group, composed of 8 partners representing 7 European countries, which stands for an ideal platform to carry out the work proposed (Figure 7). The different tasks planned in this project would not be feasible without the contribution of each of the partners, which have been specifically selected for the execution of the tasks, according to the needs of DRIvE. 


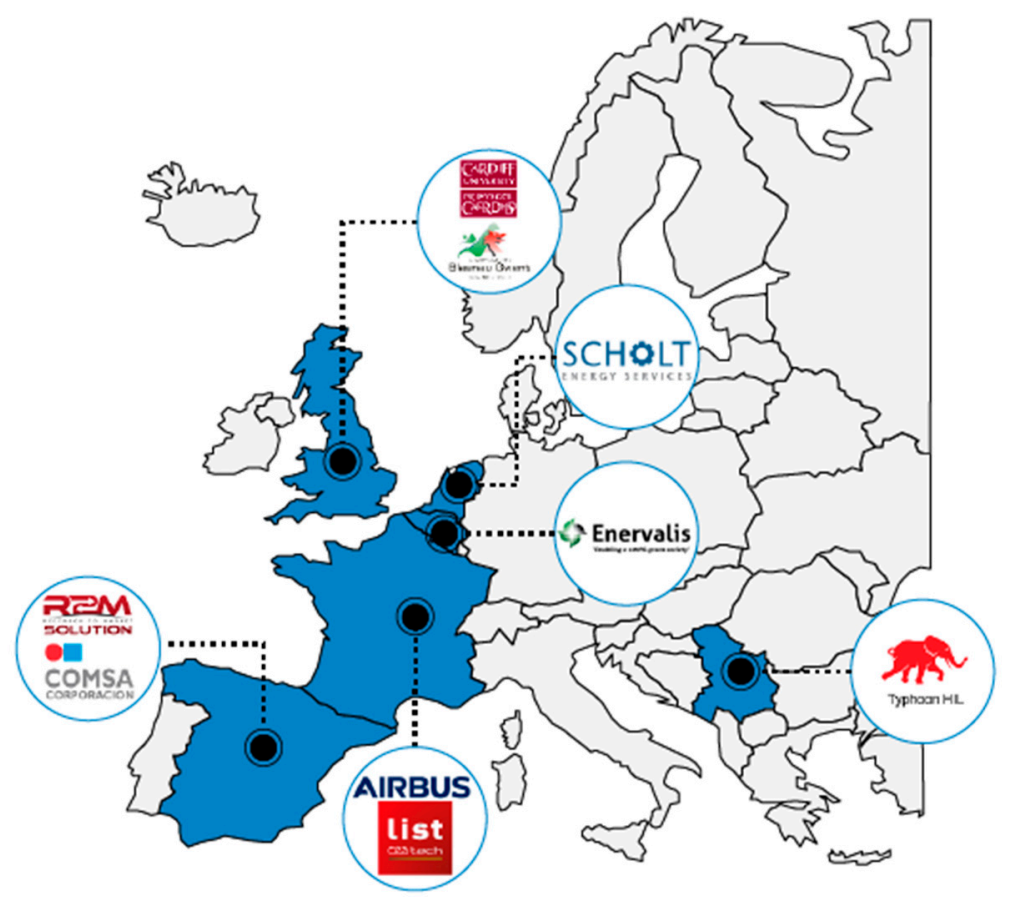

Figure 7. DRIvE consortium.

\section{Conclusions}

DRIvE will enable and boost building and district flexibility management by enhancing Demand Response capabilities of grid users, thus unlocking new businesses and services and paving the way to a fully deployed DR market in the distribution network.

Funding: This research was funded by the European Union's Horizon 2020 research and innovation programme under Grant Agreement \# 774431.

\section{References}

1. DRIvE Project in CORDIS Website. Available online: https://cordis.europa.eu/project/rcn/211957_en.html (accessed on 24 July 2018).

2. DRIvE Website. Available online: https://www.h2020-drive.eu/ (accessed on 24 July 2018). 